\title{
Correction to: Investigating Trends of Incidence Rates of Esophageal Cancer Divided by Squamous Cell Carcinoma and Adenocarcinoma in Southern Iran: a 10-Year Experience
}

\author{
Mahnaz Hosseini-Bensenjan ${ }^{1,2} \cdot$ Hossein Molavi Vardanjani ${ }^{3} \cdot$ Sezaneh Haghpanah $^{2} \cdot$ Zahra Khosravizadegan $^{4}$. \\ Kamran Bagheri-Lankarani ${ }^{5}$
}

Accepted: 4 January 2022 / Published online: 31 January 2022

(c) Springer Science+Business Media, LLC, part of Springer Nature 2022

\section{Correction to: Journal of Gastrointestinal Cancer (2021) https://doi.org/10.1007/s12029-021-00764-1}

Publisher's Note Springer Nature remains neutral with regard to jurisdictional claims in published maps and institutional affiliations.

The original version of this article, published on 2 December 2021, the authors have found an error in the published version of the paper. Dr Sezaneh Haghpanah affiliations should be "Hematology Research Center, Shiraz University of medical Sciences, Shiraz, Iran." Corrected affiliations are reflected in this correction article.

Original article has been corrected.

The original article can be found online at https://doi.org/10.1007/ s12029-021-00764-1.

Kamran Bagheri-Lankarani

lankaran@sums.ac.ir

1 MPH Department, School of Medicine, Shiraz University of Medical Sciences, Shiraz, Iran

2 Hematology Research Center, Shiraz University of Medical Sciences, Shiraz, Iran

3 MPH Department, School of Medicine, Research Center for Traditional Medicine and History of Medicine, Shiraz University of Medical Sciences, Shiraz, Iran

4 Shiraz Population-Based Cancer Registry, Shiraz University of Medical Sciences, Shiraz, Iran

5 Health Policy Research Center, Institute of Health, Shiraz University of Medical Sciences, 71348-45794 Shiraz, Iran 